\title{
Nobel Lecture: The discovery of polyacetylene film-the dawning of an era of conducting polymers*
}

\author{
Hideki Shirakawa \\ University of Tsukuba, Tsukuba, Ibaraki 305-8577, Japan
}

(Published 20 September 2001)

\begin{abstract}
The Nobel Prize in Chemistry 2000 was awarded for our discovery and development of conducting polymers, but that discovery only happened after much work on polyacetylene. In this lecture, I would like to talk about the early investigations that preceded and eventually led to the discovery of chemical doping. I do hope my talk will be of use for you, the audience, to deepen your understanding of what had happened before and how we arrived at the idea of chemical doping.
\end{abstract}

\section{PROLOGUE}

It has been recognized for many years that a very long linear conjugated polyene might have various interesting properties, especially optical, electrical, and magnetic properties. A polyene is an even number of methyne (= $\mathrm{CH}-)$ groups, covalently bonded to form a linear carbon chain bearing one $\pi$ electron on each carbon atom. Therefore the chemical structure of the polyene is best represented by the formula $\mathrm{H}(\mathrm{CH}=\mathrm{CH})_{n} \mathrm{H}$, where $n$ denotes the number of repeating units. Recently the word polyacetylene has become more popular than polyene because polyacetylene synthesized by the polymerization of acetylene has been used extensively as a specimen for various studies.

Pople and Walmsley (1962) stated in their article that "Although it is not possible to synthesize very long polyenes (polyacetylene) at present, general interest in conjugated polymers with related, but rather more complex, structures makes a full study of the electronic states of this simple polymer worthwhile." The first polymerization of acetylene was reported as early as 1958 by Natta and co-workers (Natta et al., 1958), who prepared polyacetylene that is structurally identical to a very long conjugated polyene, but that work was not accepted widely in the field. Before that time, interest in this compound was limited to theoretical attempts by chemists to explain a redshift of the absorption maximum (bathochromic effect) and an increase in the absorption coefficient (hyperchromic effect) with an increase in number of repeating units of the conjugation, and attempts by physicists to elucidate bond alternation induced by an electron-phonon interaction. A combination of experimental observations on relatively short polyenes (Kuhn, 1937; Bohlmann and Manhardt, 1956; Sondheimer et al., 1961; Nayler and Whiting, 1965; Takeuchi et al., 1973; Knoll and Schrock, 1989) and theoretical considerations, such as the free-electron model and simple Hückel molecular orbital treatments, strongly suggested that the

\footnotetext{
*The 2000 Nobel Prize in Chemistry was shared by Alan J. Heeger, Alan G. MacDiarmid, and Hideki Shirakawa. This lecture is the text of Professor Shirakawa's address on the occasion of the award.
}

difference between lengths of double and single bonds decreases with increasing conjugation and that all bonds tend to be equal in length in an infinitely long polyene. In other words, one would expect infinitely long onedimensional $\pi$ electrons to form a half filled band, or the highest-occupied (HO) and the lowest-unoccupied (LU) $\pi$-electron bands to merge with each other, leading to metallic behavior (Kuhn, 1948; Bayliss, 1952). In the 1950 s, however, it became theoretically clear that a polyene with bond alternation is energetically more stable than one with equidistant bonds (Ooshika, 1957; Longuet-Higgins and Salem, 1959; Tsuji et al., 1960; Pople and Walmsley, 1962). Since two geometrical isomers, trans and cis, are possible for each double bond, two isomeric forms, all-trans and all-cis, are expected for the two extremes of polyacetylene isomers as shown in Fig. 1. Experimentally the carbon-carbon bond lengths in polyacetylene were directly measured by Yannoni and Clarke (1983) with the use of nutation NMR spectroscopy. They obtained bond lengths of 1.36 and $1.44 \AA$ for double and single bonds, respectively, in trans form and $1.37 \AA$ for the double bond in cis form.

Even after the first synthesis by Natta and co-workers (1958), polyacetylene remained for some time a material of interest only to organic chemists (Luttinger, 1962; Daniels, 1964) and polymer chemists (Hatano et al., 1961; Watson et al., 1961; Berets and Smith, 1968) because the product was obtained as insoluble and infusible powders.

\section{II. trans OR cis}

Among a few chemists, S. Ikeda and his co-workers had been studying a mechanism of acetylene polymerization in connection with olefin polymerization by various Ziegler-Natta catalysts. They found that polymerization yields not only highly polymerized polyacetylene but also benzene, which is a cyclic trimer of acetylene, and that the ratio of these two products depends upon the species of Ziegler-Natta catalyst employed. They also observed the formation of alkylbenzenes as a minor by-product of acetylene polymerization, with a catalyst system composed of titanium tetrachloride and trialkylaluminum. In a series of experiments using carbon-14 


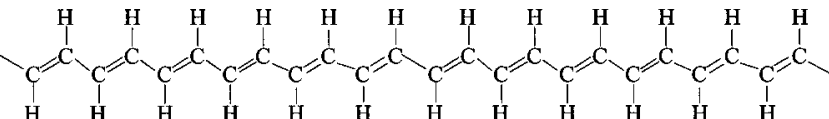

$$
\begin{aligned}
& \text { (1) }
\end{aligned}
$$

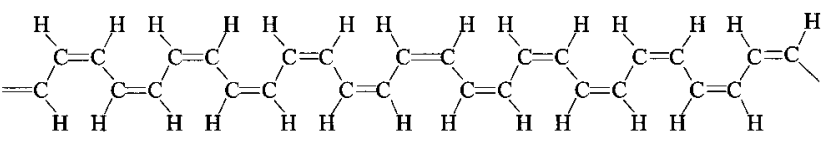

$$
\begin{aligned}
& \text { (2) }
\end{aligned}
$$

FIG. 1. All-trans (1) and all-cis (2) polyacetylene.

and deuterium, they noted that the ethyl group is introduced in the ethylbenzene when triethylaluminum labeled with carbon-14 or deuterium is used as the cocatalyst (Ikeda and Tamaki, 1966). In other experiments, in which polyacetylene was oxidized by alkaline potassium permanganate, they observed the formation of propionic and acetic acids that are derived from the alkyl groups in trialkylaluminum used as the cocatalyst (Ikeda and Tamaki, 1966). From these results, they concluded that polyacetylene and benzene could be formed from the same active site of the catalyst system. Thus the reaction proceeds by cis opening of the triple bond in acetylene followed by a cis insertion into the titaniumalkyl bond of the catalyst. This mechanism fits the orbital interaction model for the role of the catalyst by Fukui and Inagaki (1975), according to which the initially formed configuration of the double bond is cis as a result of favored orbital interaction between the inserting acetylene and the active site of the catalyst. Whether cyclic trimerization occurs to give benzene or polymerization and proceeds to give polyacetylene is determined by the conformation of the growing chain, which takes either cisoid or transoid structure at the vicinity of the active site of the catalyst (Ikeda, 1967). As no cis form had been known until then, an important question remained, namely, why the mechanism is capable of yielding only trans configuration of the double bonds in polyacetylene (Natta et al., 1958; Berets and Smith, 1968).

\section{DISCOVERY OF FILM SYNTHESIS}

The conventional method of polymerization in the laboratory requires the catalyst solution to be stirred thoroughly in order to carry out the reaction under homogeneous conditions. Acetylene polymerization was no exception. It had become customary for polymer chemists who synthesized polyacetylene to bubble acetylene gas into a catalyst solution with stirring. As a matter of fact, the product was obtained as an intractable black powder, from which it was very difficult to make specimens in a shape suitable for measurement of various spectra and properties because of its insolubility and infusibility.

Soon after I joined Ikeda's group, we succeeded in synthesizing polyacetylene directly in a form of thin film (Ito et al., 1974) by a fortuitous error in 1967. After a series of experiments to reproduce the error, we noticed that we used a concentration of the Ziegler-Natta cata-

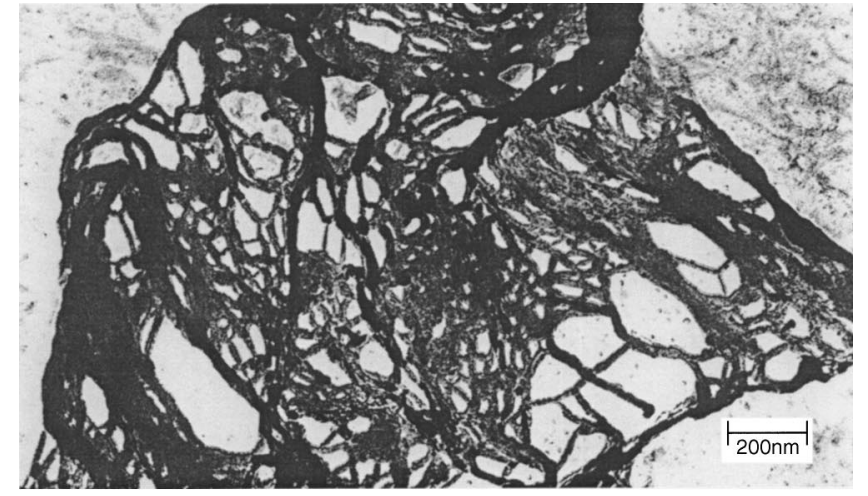

(a)

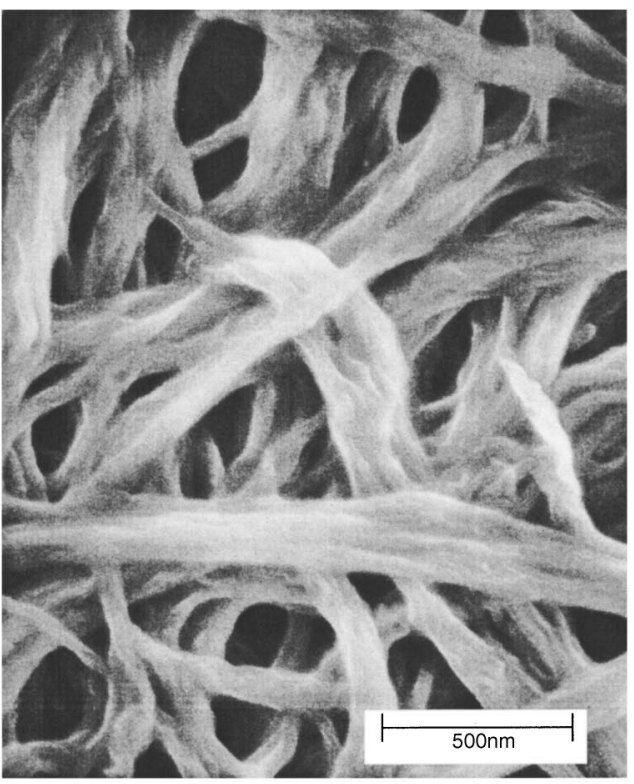

FIG. 2. Composition of polyacetylene film: (a) Transmission electron micrograph of an extremely thin film of polyacetylene; (b) scanning electron micrograph of the surface of a thick polyacetylene film.

lyst nearly a thousand times as large as that usually used. It is worth noting that the insolubility of polyacetylene contributes to the formation of film. In addition, it was found that the film is composed of entangled microfibers called fibrils; this was determined by a transmission electron microscope observation of an extremely thin film and by a scanning electron microscope observation on a surface of a thick film, as shown in Fig. 2. The fibril diameter is in the range of $20-100 \mathrm{~nm}$, depending upon the polymerization conditions. These inherent properties of polyacetylene are absolutely necessary for the film formation even under higher concentrations of the catalyst. One more important factor that should be added is that the catalyst used at that time, $\mathrm{Ti}\left(\mathrm{O}-n-\mathrm{C}_{4} \mathrm{H}_{9}\right)_{4}-\left(\mathrm{C}_{2} \mathrm{H}_{5}\right)_{3} \mathrm{Al}$, is a quite unique one from the viewpoint of its good solubility in organic solvents such as hexane or toluene to give a homogeneous solution and its high activity to give exclusively high molecular weight and crystalline polymers. By contrast, most Ziegler-Natta catalysts form precipitates that give an inhomogeneous solution when a titanium compound is mixed with alkylaluminum. 


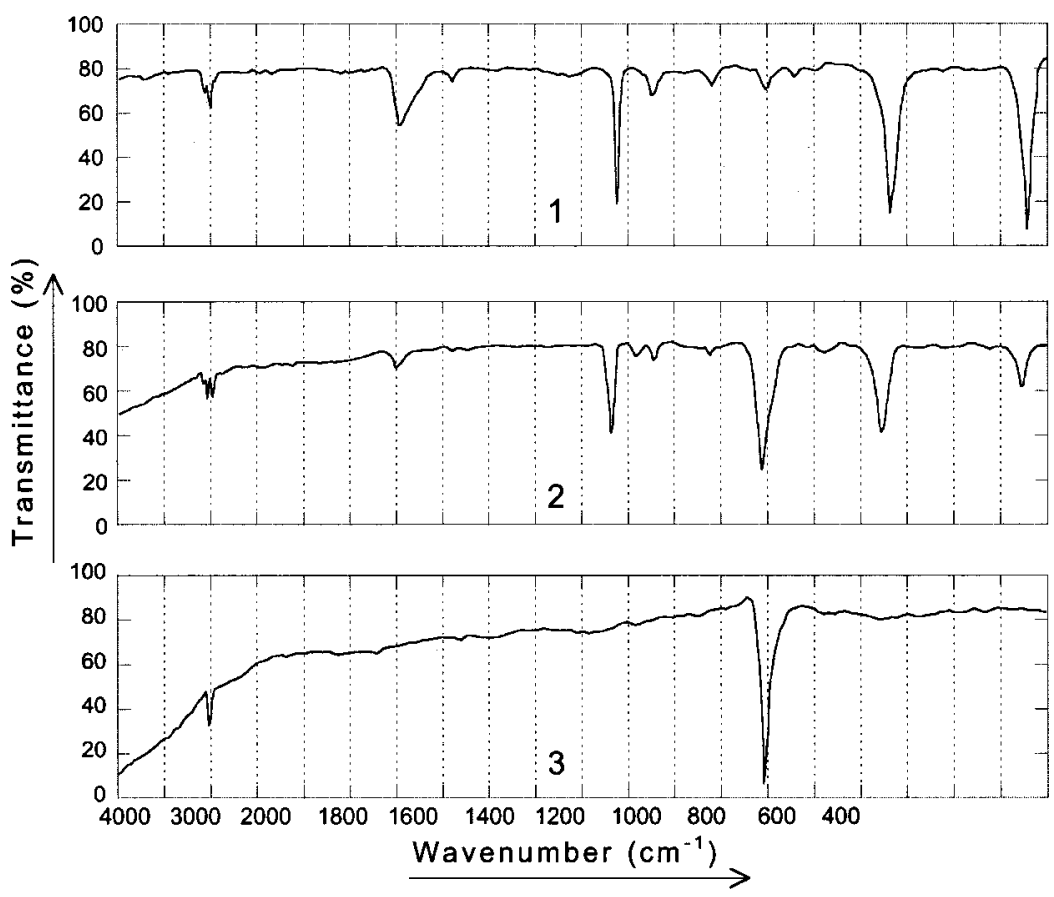

FIG. 3. Infrared spectra of polyacetylene synthesized at (1) $-78^{\circ} \mathrm{C}$, (2) $20^{\circ} \mathrm{C}$, and (3) $150{ }^{\circ} \mathrm{C}$. After Shirakawa and Ikeda (1971), Fig. 4.

The use of the films enabled us to obtain clear infrared spectra, as shown in Fig. 3 (Shirakawa and Ikeda, 1971), and analysis of these spectra indicated that the configuration of the double bonds strongly depends on the temperature of polymerization. The trans content of polyacetylene prepared by Ziegler-Natta catalysts decreases with decreasing polymerization temperature, as shown in Table I. A thermal study by Ito et al. (1975) indicated that irreversible isomerization of the cis form occurs at temperatures higher than $145^{\circ} \mathrm{C}$ to give a trans form. Thus the cis form is thermodynamically less stable than the trans one. The observed cis-rich polyacetylene synthesized at lower temperatures suggested the cis opening of the triple bond of an acetylene monomer. When the polymerization was carried out at higher temperatures, spontaneous isomerization of the growing cis double bonds occurred to give trans ones, consistent with the cis opening mechanism proposed by Ikeda (1967). The cis opening mechanism has been supported by a nutation NMR study (Yannoni and Clarke, 1983) and by the infrared study of copolymers of acetylene

TABLE I. The trans contents of polyacetylene prepared at different temperatures. Catalyst: $\mathrm{Ti}\left(\mathrm{O}-n-\mathrm{C}_{4} \mathrm{H}_{9}\right)_{4}-\left(\mathrm{C}_{2} \mathrm{H}_{5}\right)_{3} \mathrm{Al}$, $\mathrm{Ti} / \mathrm{Al}=4,[\mathrm{Ti}]=10 \mathrm{mmol} / \mathrm{l}$.

\begin{tabular}{rc}
\hline \hline Temperature $\left({ }^{\circ} \mathrm{C}\right)$ & trans content $(\%)$ \\
\hline 150 & 100.0 \\
100 & 92.5 \\
50 & 67.6 \\
18 & 40.7 \\
0 & 21.4 \\
-18 & 4.6 \\
-78 & 1.9 \\
\hline \hline
\end{tabular}

and acetylene- $d_{2}$ (Shirakawa and Ikeda, 1971). In conclusion, the open problem of why only trans polyacetylene had been known was solved by the use of films.

\section{ELECTRICAL PROPERTIES OF POLYACETYLENE FILMS}

The electrical resistivity of films obtained with various cis/trans contents by the above preparation was measured by the conventional two-probe method under vacuum in a temperature range of $-120-20{ }^{\circ} \mathrm{C}$ (Shirakawa et al., 1978). The resistivity and energy gap of trans-rich polyacetylene were $1.0 \times 10^{4} \Omega \mathrm{cm}$ and 0.56 $\mathrm{eV}$, respectively, whereas the values of a cis-rich $(80 \%)$ one were $2.4 \times 10^{8} \Omega \mathrm{cm}$ and $0.93 \mathrm{eV}$, respectively. Hatano and co-workers (Hatano et al., 1961) reported that the resistivity and energy gap measured on compressed pellets of powder polyacetylene synthesized by the same catalyst system were in the range of $1.4 \times 10^{4}$ and $4.2 \times 10^{5} \Omega \mathrm{cm}$, and $0.46 \mathrm{eV}$, respectively, in good agreement with those for trans-rich polyacetylene film. In conclusion, it became apparent that the intrinsic electrical properties do not change very much between powder and film.

\section{HALOGENATION OF POLYACETYLENE FILMS}

Since no improvement in electrical conductivity was observed in the films as prepared, we tried to convert them to graphite, which we thought might be possible due to the high carbon content (as much as $92.3 \%$ ). We first tried heating the films. Differential thermal analysis of cis-rich polymer revealed the existence of two exothermic peaks at 145 and $325^{\circ} \mathrm{C}$ and one endothermic 


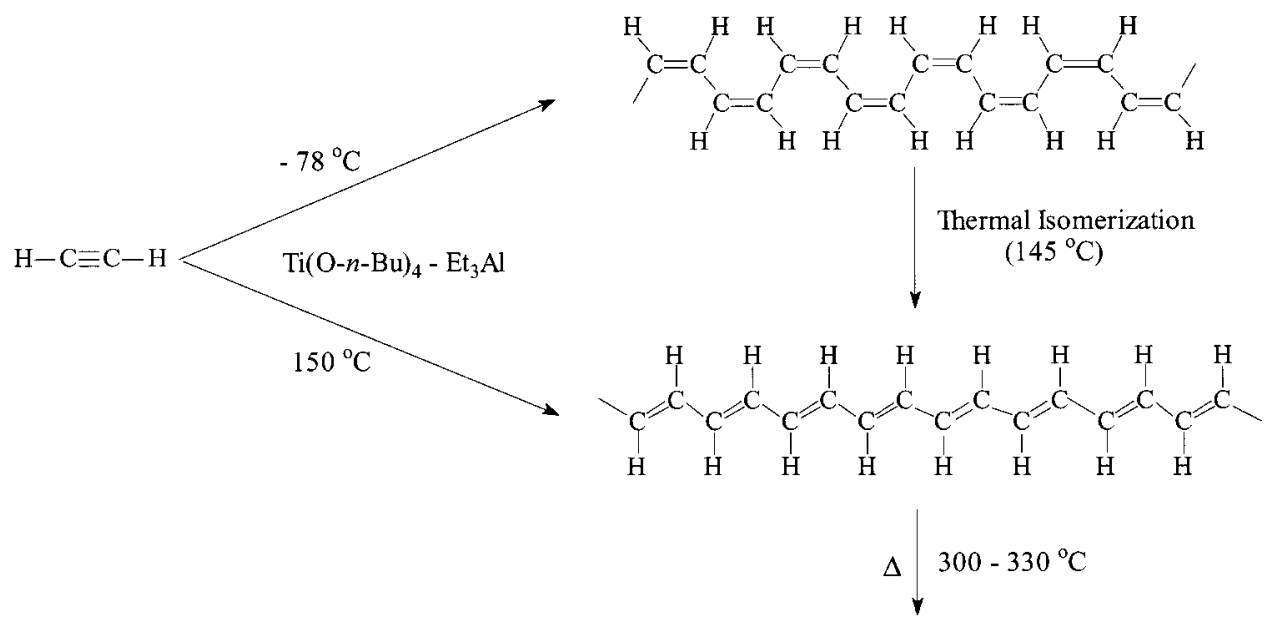

$\mathrm{C}_{1}-\mathrm{C}_{8}$ Hydrocarbons $60 \%$ Brown Oily Paste $\quad 40 \%$ $\frac{\text { Themal Decomposition }}{>420^{\circ} \mathrm{C}}$
Crosslinked Brown Polymer (No longer conjugated double bonds)

FIG. 4. Thermal characteristics of polyacetylene.

peak at $420^{\circ} \mathrm{C}$, which were assigned to cis-trans isomerization, hydrogen migration accompanied by a crosslinking reaction, and thermal decomposition, respectively (Ito et al., 1975), as shown in Fig. 4. Thermogravimetric analysis showed that weight loss reached $63 \%$ at $420{ }^{\circ} \mathrm{C}$. Therefore pyrolysis of the polyacetylene films is not suitable for such purposes.

Another approach to removing the hydrogen is to use a halogen reaction. Electrophilic addition of halogens such as chlorine and bromine to a carbon-carbon double bond is a well-known reaction and proceeds with good yield and fewer side reactions. Elimination of hydrogen halide from adjacent carbon atoms in a halogenated hydrocarbon is also a well-known reaction to introduce a carbon-carbon double or triple bond. Since polyacetylene reacts with chlorine and bromine at mild conditions, we thought carbonization might be possible by combining both reactions. Thus a polyacetylene film was reacted with chlorine at room temperature to give a chlorinated polyacetylene film, which was subsequently treated with a basic reagent to eliminate hydrogen chloride to give a carbon film in a satisfactory yield. However, we found that the carbon film was hardly graphitized even when heated at $2000^{\circ} \mathrm{C}$ for several hours.

Fully chlorinated polyacetylene has a chemical structure of $(\mathrm{CHCl}-\mathrm{CHCl})_{n}{ }^{-}$, corresponding to a polymer of 1,2-dichloroethylene. It is known, however, that 1,2disubstituted ethylene is very difficult to polymerize. Therefore the chlorination of polyacetylene is the sole method for synthesizing poly(1,2-dichloroethylene). In order to investigate the chlorination process of polyacetylene film, in situ measurements were planned to obtain infrared spectra during chlorination of polyacetylene film.

It is well known that chlorination of the simplest conjugated polyene, 1,3-butadiene, gives two isomeric products, 1,2-addition product (3,4-dichloro-1-butene) and 1,4-addition product (1,4-dichloro-2-butene). Likewise, 1,3,5-hexatriene gives three isomers, 1,2-, 1,4-, and 1,6addition products. The reason why this reaction gives various products can be traced to the existence of different resonance structures in the intermediate, as shown in Fig. 5. The third isomer is thermodynamically the most stable because the molecule has an inner conjugated diene structure with the largest energy of resonance stabilization.

The motivation of the study was to confirm that a $1,2 n$ addition of chlorine might occur at the initial step of the reaction, as shown in Fig. 6. Since the longer inner polyene structure has the larger energy of resonance stabilization, it is anticipated that products of a $1,2 n$ addition with larger $n$ predominate rather than a 1,2 addition product at the initial step of the reaction or in the partially chlorinated polyacetylene. If such a structure could be detected by infrared spectroscopy, one might expect that the intermediate carbocation would be able to migrate far away from the reaction site through the conjugated polyene structure.

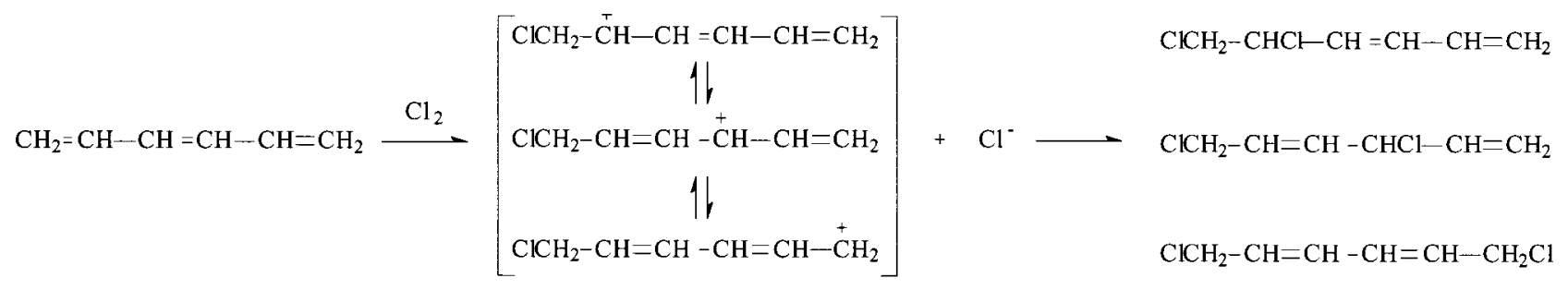

FIG. 5. Possible resonance structures in the intermediate during the process of the chlorination of 1,3,5-hexatriene and three chlorinated products. 

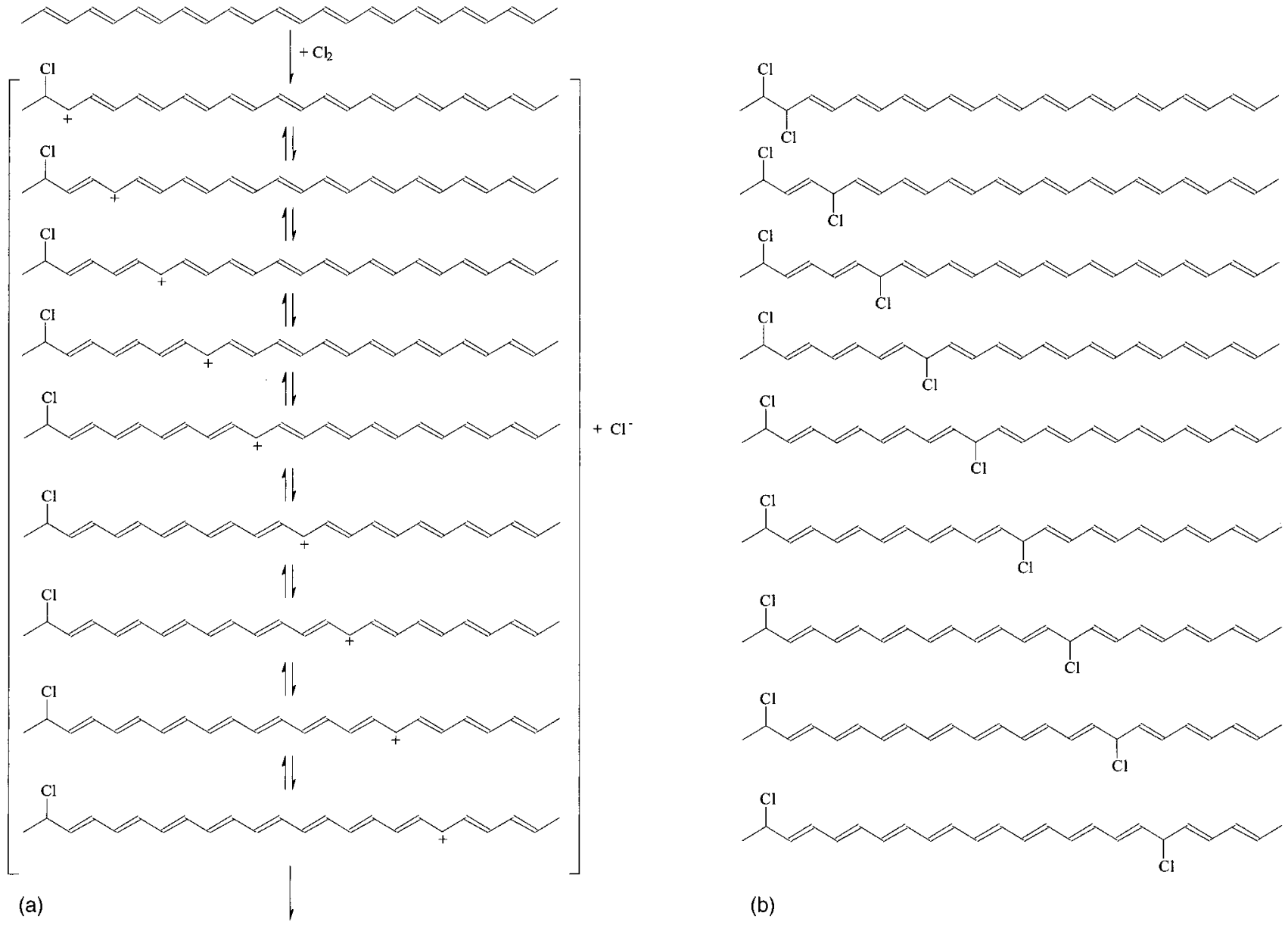

(b)

FIG. 6. (a) Possible resonance structures in the intermediate in the chlorination of polyacetylene. (b) Expected chemical structures of partially chlorinated polyacetylene.

Contrary to this expectation, when a trace amount of chlorine was added to the film, it did not produce a line spectrum. Instead, the infrared spectrophotometer recorded a complete absorption in the full range of wavelengths from $4000-400 \mathrm{~cm}^{-1}$. The polyacetylene film had changed into an opaque material. Upon continued reaction with additional chlorine, the spectrum became clearer, to give that corresponding to a chlorinated polyacetylene.

\section{EPILOGUE}

We know now that the opacity was due to the socalled "doping-induced infrared band," actually composed of three bands at 1397, 1288, and $888 \mathrm{~cm}^{-1}$ (Fincher et al., 1979; Rabolt et al., 1979; Harada et al., 1980), which have extremely strong absorption coefficients compared with those of the undoped polymer. The observed isotope shifts of these bands in the spectra of poly $\left(\right.$ acetylene- ${ }^{13} \mathrm{C}_{2}$ ) and poly (acetylene- $\left.d_{2}\right)$ demonstrated that these bands were of vibrational origin (Harada et al., 1980) in the vicinity of the carbocation or positively charged carbon in the conjugated polyene. At present, the carbocation formed in long conjugated polyenes is widely known as a positively charged soliton (Su et al., 1979) that acts as a charge carrier for electrical conduction. At that time, to my regret, I did not recognize that this carbocation could be a charge carrier and thus polyacetylene could be the first conducting polymer. To open an era of conducting polymers, we had to wait until we intentionally carried out the doping experiment with the use of bromine on Tuesday November 23rd, 1976 at the University of Pennsylvania, and successively with iodine (Chiang et al., 1977; Shirakawa et al., 1977).

\section{ACKNOWLEDGMENTS}

I want to express my heartfelt thanks to the late Sakuji Ikeda, who gave me a chance to work on acetylene polymerization, to Masahiro Hatano, who contributed to the semiconducting polymers and encouraged me in my work, to the late Shu Kambara, who organized our polymer research group and supported us in our work even after his retirement, and to Hyung Chick Pyun, with whom I shared the discovery of polyacetylene film by fortuitous error. Takeo Ito was the first graduate student to work with me, who made a great contribution at the initial stage of this work. My special thanks go to Shiro Maeda, Takehiko Shimanouchi, and Mitsuo Tasumi for helpful discussions on the vibration analyses study. I am indebted to Shigeru Ando for mea- 
surements of solid-state properties and to Yoshio Sakai for electrical conductivity measurements. Throughout this period our research was partly supported by Grantsin-Aid for Scientific Research from the Ministry of Education, Culture and Science of Japan.

\section{REFERENCES}

Bayliss, N. S., 1948, J. Chem. Phys. 16, 287.

Bayliss, N. S., 1952, Q. Rev., Chem. Soc. 6, 319.

Berets, D. J., and D. S. Smith, 1968, Trans. Faraday Soc. 64, 823.

Bohlmann, F., and H. Manhardt, 1956, Chem. Ber. 89, 1307.

Chiang, C. K., C. R. Fincher, Jr., Y. W. Park, A. J. Heeger, H. Shirakawa, E. J. Louis, S. C. Gau, and A. G. MacDiarmid, 1977, Phys. Rev. Lett. 39, 1098.

Daniels, W. E., 1964, J. Org. Chem. 29, 2936.

Fincher, C. R., Jr., M. Ozaki, A. J. Heeger, and A. G. MacDiarmid, 1979, Phys. Rev. B 19, 4140.

Fukui, K., and S. Inagaki, 1975, J. Am. Chem. Soc. 96, 4445.

Harada, I., Y. Furukawa, M. Tasumi, H. Shirakawa, and S. Ikeda, 1980, J. Chem. Phys. 73, 4746.

Hatano, M., S. Kambara, and S. Okamoto, 1961, J. Polym. Sci. 51, S26.

Ikeda, S., 1967, Kogyo Kagaku Zasshi 70, 1880.

Ikeda, S., and A. Tamaki, 1966, J. Polym. Sci., Polym. Phys. Ed. 4, 605.

Ito, T., H. Shirakawa, and S. Ikeda, 1974, J. Polym. Sci., Polym. Chem. Ed. 12, 11.

Ito, T., H. Shirakawa, and S. Ikeda, 1975, J. Polym. Sci., Polym. Chem. Ed. 13, 1943.
Knoll, K., and R. R. Schrock, 1989, J. Am. Chem. Soc. 111, 7989.

Kuhn, H., 1948, Helv. Chim. Acta 31, 1441.

Kuhn, R., 1937, Angew. Chem. 34, 703.

Longuet-Higgins, H. C., and L. Salem, 1959, Proc. R. Soc. London, Ser. A 252, 172.

Luttinger, L. B., 1962, J. Org. Chem. 27, 1591.

Natta, G., G. Mazzanti, and P. Corradini, 1958, Atti Accad. Naz. Lincei, Cl. Sci. Fis., Mat. Nat., Rend. 25, 2.

Nayler, J. H. C., and M. C. Whiting, 1965, J. Chem. Soc. 1965, 3037.

Ooshika, Y., 1957, J. Phys. Soc. Jpn. 12, 1238; 12, 1246.

Pople, J. A., and S. H. Walmsley, 1962, Mol. Phys. 5, 15.

Rabolt, J. F., T. C. Clarke, and G. B. Street, 1979, J. Chem. Phys. 71, 4614.

Shirakawa, H., and S. Ikeda, 1971, Polym. J. (Tokyo) 2, 231.

Shirakawa, H., T. Ito, and S. Ikeda, 1978, Makromol. Chem. 179, 1565.

Shirakawa, H., E. J. Louis, A. G. MacDiarmid, C. K. Chiang, and A. J. Heeger, 1977, J. Chem. Soc. Chem. Commun. 1977, 578.

Sondheimer, F., D. A. Ben-Efraim, and R. Wolovsky, 1961, J. Am. Chem. Soc. 83, 1675.

Su, W. P., J. R. Schrieffer, and A. J. Heeger, 1979, Phys. Rev. Lett. 42, 1698.

Takeuchi, Y., A. Yasuhara, S. Akiyama, and M. Nakagawa, 1973, Bull. Chem. Soc. Jpn. 46, 822.

Tsuji, M., S. Fuzinaga, and T. Hashino, 1960, Rev. Mod. Phys. 32, 425 .

Watson, W. H., Jr., McMordie, Jr., and L. G. Lands, 1961, J. Polym. Sci. 55, 137.

Yannoni, C. S., and T. C. Clarke, 1983, Phys. Rev. Lett. 51, 1191. 